\title{
Load movement control system for rollover risk reduction of tanker trucks
}

\section{A. Cammarata, G. La Rosa, A. Pellegrino* and A. Risitano}

Dipartimento di Ingegneria Industriale e Meccanica, Università degli studi di Catania,

Viale Andrea Doria 6,

95125 Catania, Italy

Fax: 39095 330258/337994

E-mail: acamma@diim.unict.it_E-mail: glarosa@diim.unict.it

E-mail: pellegrino_anto79@yahoo.it

E-mail: arisitan@diim.unict.it

${ }^{*}$ Corresponding author

\begin{abstract}
In this paper a proposal for a control system that provides liquid cargo movement from one side to another is made. The system acts to minimise the effects of lateral load transfer and fluid oscillation. This consists of a main tank, two lateral tanks, regulators and a set of electropumps effecting the movement of the fluid. According to literature, in order to assess the risk of rollover, a normalised lateral load transfer has been used. Simulations of the lateral load transfer response have also been carried out to assess the improvements in lateral stability of the vehicle.
\end{abstract}

Keywords: rollover; fluid sloshing; partially filled tankers; Simulink; load transfer.

Reference to this paper should be made as follows: Cammarata, A., La Rosa, G., Pellegrino, A. and Risitano, A. (2011) 'Load movement control system for rollover risk reduction of tanker trucks', Int. J. Heavy Vehicle Systems, Vol. 18, No. 3, pp.303-321.

Biographical notes: Alessandro Cammarata received his $\mathrm{MSc}$ and $\mathrm{PhD}$ in Structural Mechanics from the Faculty of Mechanical Engineering, University of Catania, in 2004 and 2008, respectively. He has been a foreign student at the Centre for Intelligent Machines of the McGill University of Montreal in 2007. Nowadays, he is under a research contract with Thales Alenia Space. His research interests deal with kinematics, dynamics modelling and optimisation of parallel robots. He is author of more than 20 scientific papers in international journals and conference proceedings.

G. La Rosa graduated in Electronic Engineering in 1978 at the University of Pisa (Italy), since 1982 he works at the University of Catania as Assistant Professor (from 1982 to 1992), Associate Professor (from 1992 to 2000) and (since 2000) Full Professor of Machine Design. He is author of more than 150 scientific papers on Italian and international reviews and conference proceedings. His research interests include, inter alia, stress-strain analysis, static and dynamic tests on traditional and innovative materials, innovative methods to predict fatigue life, design of biomechanical systems, eco-design, integrated product design. 
Antonio Pellegrino received his Master's Degree in Mechanical Engineering from the University of Catania (Italy) in 2008 discussing a thesis on the rollover of partially filled tanker trucks and the development of an anti-rollover system. He worked as collaborator of the University of Catania on the development of an automated tank cleaning (desludging) and hydrocarbons recovery system for oil storage tanks. $\mathrm{He}$ is a $\mathrm{PhD}$ student in Structural Mechanics at the University of Catania.

A. Risitano graduated in Mechanical Engineering at the Polytechnic of Turin, Italy, July 1969. He has been Assistant Professor of Machine Design and Calculation at the Polytechnic of Turin, Mechanical Engineering (1971-1980), Since 1 November, 1980 he is full professor of Machine Design at the University of Catania. He is author of more than 150 scientific papers and owner of 6 patents including a thermographic method for rapid determination of the fatigue limit. His research interests include, inter alia, static and dynamic characterisation of materials, criteria for sustainable design, analysis of surface cracks modelling and control of automotive systems.

\section{Introduction}

In most cases of transport, involving the use of heavy vehicles on the road, the truck cargo is fixed and centred with respect to the longitudinal axis of the vehicle. In some cases, however, the load may have possibilities of movement inside the vehicle both transverse and longitudinal directions, conditioning cornering performance and rollover safety (Winkler, 2000). The most important and common case of moving cargo is the one regarding transport of liquids within tanker trucks. A partially filled tanker truck allows the liquid to move from one side to the other producing a 'sloshing' load condition and affecting the rollover propensity of the vehicle. The oscillation of liquid regards vehicle safety because of the lateral shift of the centre of gravity (CG) that reduces the lateral acceleration threshold causing rollover. In fact, rollover occurs when the resultant of transverse forces becomes external to the support polygon formed by the central points of tyres-road contact areas. When a tanker truck is cornering, the centrifugal force increases its effect because of the liquid motion, further increasing the height and the lateral shift of the CG (on the opposite side with respect to the centre of curvature) more than in the case of fixed load. It is also necessary to consider that liquid motion can interact with lateral movements of the vehicle in such a way as to amplify the magnitude of oscillations until it becomes excessive, further reducing the rollover threshold. The aim of the study reported in this paper is the dynamics analysis of lateral fluid oscillations for partially filled tanker trucks and the development of an active-passive system designed to minimise lateral instability. This analysis has been modelled by means of Matlab/Simulink and pertains to vehicles used for the transport of dangerous goods.

There are many papers concerning rollover prevention or rollover risk reduction systems for heavy vehicles, or generally, for vehicles with an elevated position of the CG (Hac, 2000). Vehicles with an elevated CG are especially threatened by rollover. In literature, the issue has been addressed with different approaches. In Sampson et al. (2000) and Sampson (2000) the roll control system consists of five anti-roll bars driven by nine hydraulic actuators. In Eisele (2000), the Vehicle Dynamics Control System 
(VDCS) uses differential breaking control to simultaneously perform three tasks: rollover prevention, side slip reduction and to make the vehicle yaw-rate match the driver's desired yaw-rate. In Odenthal et al. (1999), a linear steering control, extended by non-linear emergency steering and braking control, is presented. The rollover avoidance control consists of three feedback loops: continuous operation steering control, emergency steering control and emergency braking control. Another approach (Gáspár et al., 2005) uses a combined control structure to decrease the rollover risk of heavy vehicle. This approach employs active anti-roll bars combined with an active brake control; the control of the active brake system is extended with a new calculation procedure in which the values of the lateral load transfer are predicted in advance, using a short time interval, to determine whether these values may exceed the critical value corresponding to the rollover.

All cited approaches achieve good results in terms of rollover avoidance acting, respectively, on brakes, steering and anti-roll bars. In this paper, a new approach is presented, acting directly on the lateral distribution of the liquid cargo transported.

\section{Conceptual system description}

The proposed rollover risk reduction system provides lateral liquid cargo movement to minimise the effects of lateral load transfer and fluid oscillation, further lowering the lateral acceleration threshold at which rollover occurs. What we want to create in the field of heavy vehicles is something conceptually similar to what you can see watching a sailing race in which the movement of the crew contributes in a decisive way to the boat balance. The concept outlined earlier is achieved, in the case of a tanker truck, by means of controlled movements of the liquid cargo. The system consists of a main tank and two smaller lateral tanks, which can change their fill level thanks to the employment of a set of electropumps, as shown in Figure 1(a). The main tank carries most of the payload passing from a total filling condition to successive partial filling conditions.

Figure 1 (a) Schematic design of the anti-rollover system and (b) trammel pendulum

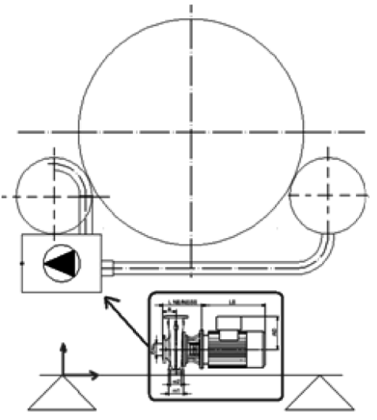

(a)

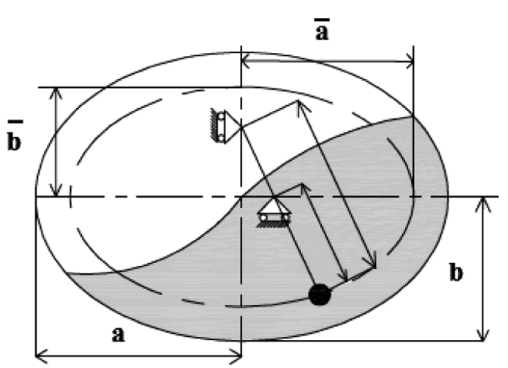

(b)

The fill-level variation of lateral tanks compensates the effects of lateral load transfer that are worsened by fluid oscillation. The liquid load transfer exclusively concerns the lateral tanks, located in a lower position compared with the position of the main tank. 
Obviously, while cornering, the inner lateral tank fill level has to be higher than the fill level relative to the opposite lateral tank. At every moment, the load carried by both the lateral tanks corresponds to the volume of one of them. Thus, the maximum transportable liquid volume is equal to the sum of the volumes of the main tank and of one lateral tank. The percent of total payload loss, in comparison with a standard tanker truck, is about $15-20 \%$.

It must be stressed that the proposed system is a passive-active system; in fact, the lateral stabilising effect is the sum of three contributions: the controlled liquid movement from one side to the other of the tanker (active contribution), the CG lowering due to the presence of lateral tanks and the braking effect due to the pumps power absorption (passive contributions).

\section{Fluid lateral sloshing simulation: normalised lateral load transfer}

The main dynamical effect of lateral sloshing is an oscillation of the liquid CG about the longitudinal axis of the tank. According to literature (Dodge, 2000; Salem, 2000; Acarman and Özgüner, 2003), the lateral sloshing dynamics can be modelled through an equivalent mechanical model. It has been found that a suitable equivalent mechanical model for partially filled elliptical tankers consists of a pendulum that follows an elliptical path, the trammel pendulum shown in Figure 1(b). Figure 1(b) also shows the fundamental antisymmetric sloshing wave. This particular wave has the lowest natural frequency $\omega_{1}$ (the natural frequency parameter $\omega_{l}(a / g)^{0.5}$ for a horizontal cylindrical tank of radius $a$ ranges from about 1.0 to about 4.0 depending on the fill level). Waves with two or more peaks and valleys, i.e., with higher natural frequencies, can also occur. The mechanical model shown in Figure 1(b) can represent these higher-order waves including an additional pendulum for each mode. Since only the fundamental antisymmetric wave creates significant forces or torques on the tank, the magnitudes of the pendulum for the higher-order waves are very small compared with the fundamental mode and, thus, higher-order modes are usually of little concern (Dodge, 2000). The equation of motion for the trammel pendulum, owing to an external lateral acceleration $\ddot{x}$, is:

$$
\left(\bar{a}^{2} \cos ^{2} \vartheta+\bar{b}^{2} \sin ^{2} \vartheta\right) \ddot{\vartheta}+\frac{1}{2}\left(\bar{b}^{2}-\bar{a}^{2}\right) \ddot{\vartheta}^{2} \sin 2 \vartheta+g \bar{b} \sin \vartheta+\bar{a} \cos \vartheta \cdot \ddot{x}=0
$$

where $\vartheta$ (Figure 2) is the angle of swing described by the pendulum.

Figure 2 Equivalent mechanical models of lateral liquid sloshing
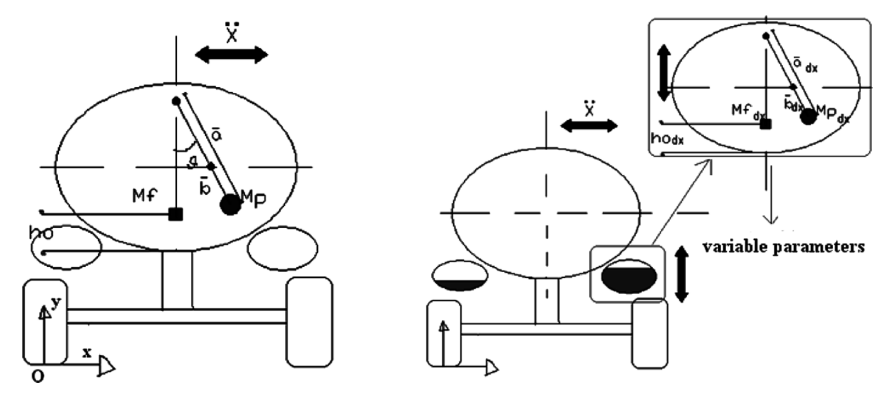
An amount of liquid mass is assumed to be fixed relative to a coordinate system attached to the tank (Figure 2). This is necessary to match the trammel pendulum model to the dynamic effects of lateral sloshing of the liquid load. Thus, to achieve a complete simulation of fluid lateral sloshing in a partially filled elliptical tank, four parameters need to be evaluated (Salem, 2000). These parameters are: the arms of the pendulum $\bar{a}$ and $\bar{b}$, the moving mass $M_{p}$ and the height $h_{0}$ at which the fixed mass $M_{f}$ is located relative to the tank base point. All the parameters exclusively depend on the normalised fill level $h / 2 b$ (see Nomenclature) and on the tank shape aspect ratio $a / b$ (Salem, 2000), i.e.:

$$
\begin{aligned}
& \frac{\bar{b}}{b}=1-\left(1.780896-\frac{1.542048}{\left(\frac{a}{b}\right)}\right) \cdot\left(\frac{h}{2 b}\right)+\left(0.7726259-\frac{1.304727}{\left(\frac{a}{b}\right)}\right) \cdot\left(\frac{h}{2 b}\right)^{2}=\Lambda \\
& \bar{a}=\bar{b} \cdot \frac{a}{b} \\
& \frac{M_{p}}{M_{t}}=1+\left[-0.863+1.237 \cdot \ln \left(\frac{a}{b}\right)\right] \cdot\left(\frac{h}{2 \cdot b}\right)-\left[0.1226+1.2489 \cdot \ln \left(\frac{a}{b}\right)\right] \cdot\left(\frac{h}{2 b}\right)^{2}=\Phi(4) \\
& M f+M p=M t \\
& \frac{h_{o}}{b}=\frac{Y_{c g}-\Phi \cdot b \cdot(1-\Lambda)}{b \cdot(1-\Phi)}
\end{aligned}
$$

where $Y_{C G}$ is the vertical coordinate of the CG of the total mass of the liquid relative to the tank base point, when calculated in static conditions.

Therefore, the forces exerted by the liquid inside the main tank, both due to lateral acceleration and due to the fluid oscillation, are expressed as

$$
\begin{aligned}
& F_{p 1, x}=-M_{p} \cdot \ddot{r}_{x}=-M_{p} \cdot\left[\bar{a} \cdot\left(-\dot{\theta}^{2} \cdot \sin \vartheta+\ddot{\vartheta} \cdot \cos \vartheta\right)+\ddot{x}\right] \\
& F_{p 1, y}=-M_{p} \cdot \ddot{r}_{y}=-M_{p} \cdot \bar{b} \cdot\left(\dot{\vartheta}^{2} \cdot \cos \vartheta+\ddot{\vartheta} \cdot \sin \vartheta\right) \\
& F_{p 2, y}=-M_{p} \cdot g \\
& F_{f, y}=-M_{f} \cdot g \\
& F_{f, x}=-M_{f} \cdot \ddot{x}
\end{aligned}
$$

where $\ddot{r}_{x}$ and $\ddot{r}_{y}$ are, respectively, the horizontal and vertical components of the acceleration acting on the moving mass of the mechanical equivalent model. The mathematical expressions of forces exerted by the liquid inside the lateral tanks are the same. However, all the parameters of the sloshing equivalent mechanical model depend on the fill level (Figure 2), therefore, a Matlab algorithm capable of updating the lateral tanks fill level, according to the flow pattern over time, has been developed and 
included into the anti-rollover system Simulink model. Liquid flow from one lateral tank to the other is controlled by a set of feedback regulators.

Forces exerted by the liquid inside the pipes linking the lateral tanks have also been taken into account including weight, centrifugal and inertial forces during the pumps shutdown or start-up transient periods. The latter are calculated considering incompressible fluid, mean flow speed and linear variation of the flow rate during the pumps transient periods (Citrini and Noseda, 1987). Weights and centrifugal forces owing to the masses of pumps and tanks tare are also included in the semitrailer model.

Once the transverse forces relative to the semitrailer longitudinal axis are calculated, right wheels and left wheels vertical loads are determined, at every instant of simulation time, from the equilibrium of vertical forces and roll moments. To evaluate the rollover risk, a normalised lateral load transfer coefficient (Odenthal et al., 1999) is defined as

$$
R=\frac{F_{z, R}-F_{z, L}}{F_{z, R}+F_{z, L}}
$$

During straight driving along a horizontal road, the 'rollover coefficient' $R$ equals zero because left and right wheels vertical loads have the same value $\left(F_{z, R}=F_{z, L}\right)$. In the case of incipient wheels lifting, $R$ takes on the value $R=-1$ (right wheels lift off) or the value $R=+1$ (left wheels lift off). The vehicle model is only valid if absolute value of $R$ does not exceed the unity, meaning that all wheels have road contact as well.

\section{Control algorithms}

The rollover risk reduction control system is composed of four feedback regulators (Bolzern et al., 2004): a threshold controller, two compensators and a final balancer of lateral tanks fill levels.

The control variables, under which the set of feedback regulators operates, are:

- The absolute value of the normalised lateral load transfer (or rollover coefficient), $|R|$

$$
|R|=\left|\frac{F_{z, R}-F_{z, L}}{F_{z, R}+F_{z, L}}\right| .
$$

- The sign of $R, \operatorname{sign}(R)$

- The external lateral acceleration, which the tanker truck is exposed to, $\ddot{x}$

- The difference between the liquid volume inside the right and left lateral tanks, $\Delta V=V_{d x}-V_{s x}$.

\subsection{Threshold controller}

The threshold controller is the main control system. While cornering, if $R$ exceeds a threshold value, this regulator determines a liquid flow to the inner tank, filling it and, consequently, emptying the outer lateral tank. Hereafter, inner and outer will be referred to the centre of curvature of the curve. 
The threshold value of $R$ is different depending on whether the external lateral acceleration is increasing or decreasing, with a lower threshold in the case of increasing lateral acceleration. This is necessary to permit a rapid system response when entering a curve and a rapid restoring of the initial condition of equality between the lateral tanks fill levels while exiting a curve. Once the liquid flow to the inner lateral tank has begun, it will continue for five seconds from the moment in which external lateral acceleration stops increasing. This is important to allow a tanker truck, equipped with the presented anti-rollover system, to negotiate a succession of reverse curves avoiding instability due to the required lateral tanks filling time. The threshold controller rules are synthetically described by the following relations:

$$
\begin{aligned}
& \text { If: } \frac{\mathrm{d}|\ddot{x}|}{\mathrm{d} t} \geq-0.1 \frac{m}{\mathrm{~s}^{3}}, \quad|R|>0.3, \Delta t_{\text {crono }}<\Delta t_{\text {crono }}^{* *} \text { then }|Q|>0 \frac{m^{3}}{\mathrm{~s}}, \\
& \operatorname{sign}(Q)=-\operatorname{sign}(R) \\
& \text { Else if: } \begin{aligned}
\frac{\mathrm{d}|\ddot{x}|}{\mathrm{d} t}<-0.1 \frac{m}{\mathrm{~s}^{3}}, \quad|R|>0.8, \quad \Delta t_{\text {crono }}<\Delta t_{\text {crono }}^{* *} \text { then }|Q|>0 \frac{\mathrm{m}^{3}}{\mathrm{~s}}, \\
\quad \operatorname{sign}(Q)=-\operatorname{sign}(R)
\end{aligned} \\
& \Delta t_{\text {crono }}^{* *}=5 \mathrm{~s}
\end{aligned}
$$

where $Q$ is the controlled flow rate and $\Delta_{\text {tcrono }}$ is the time elapsed from the moment in which external lateral acceleration stops increasing. Flow rate is assumed to be positive if directed towards the right lateral tank and negative if directed towards the left lateral tank.

It should be noted that in equations (14) and (15) the threshold for the time derivative of the acceleration or 'jerk' is -0.1 and not zero. Otherwise, during a constant radius curve, the braking effect, due to the pumps power absorption, would stop the action of the threshold controller.

\subsection{Compensators}

Compensators role is to provide the initial restoring of the equality between the lateral tanks fill levels. The two compensators operate when, through the simultaneous verification of the input signals coming from various sensors, the road curvature is decreasing. Assuming, for example, to negotiate a right-hand curve, the threshold controller action provides the filling of the inner lateral tank, i.e., the right tank that, consequently, causes the emptying of the opposite left lateral tank. During the decreasing radius transition curve at the end of the right-end curve, compensator 1 checks if the threshold controller stopped its action. It also checks whether lateral acceleration is decreasing and whether right tank fill level is higher than left tank fill level. If all the conditions are verified, the restoring phase of initial fill levels begins and the compensator commands the electropumps to transfer a flow rate to the left lateral tank. The compensators control laws are schematically described through the following relations: 


\section{Compensator 1}

$$
\text { If: } \operatorname{sign}(R)<0 \text { or } 0 \leq R \leq 0.3, \quad Q_{\text {threshold }}=0 \frac{m^{3}}{s}, \frac{\mathrm{d} \ddot{x}}{\mathrm{~d} t}<-0.05 \frac{m}{\mathrm{~s}^{3}}, \quad \Delta V>0 m^{3}
$$

then $Q<0 \mathrm{~m}^{3} / \mathrm{s}$ (to the left lateral tank)

Compensator 2

$$
\text { If : } \operatorname{sign}(R)>0 \text { or }-0.3 \leq R \leq 0, \quad Q_{\text {treshold }}=0 \frac{m^{3}}{s}, \quad \frac{\mathrm{d} \ddot{x}}{\mathrm{~d} t}>0.05 \frac{\mathrm{m}}{\mathrm{s}^{3}}, \quad \Delta V<0 \mathrm{~m}^{3}
$$

then $Q>0 \mathrm{~m}^{3} / \mathrm{s}$ (to the right lateral tank)

where $Q_{\text {threshold }}$ is the liquid flow commanded by the threshold controller. Note that lateral acceleration is assumed to be positive while cornering on the right, whereas it is assumed negative during a left-hand curve. Likewise threshold controller, the threshold for the time derivative of the acceleration or 'jerk' is not zero. This is necessary to avoid that compensators start their action too early, when road curvature has not decreased enough. Since the action of the threshold controller relates to safety, a more limiting condition on the decreasing of lateral acceleration is there imposed.

Compensators action is fundamental because it allows the system to react promptly to a succession of reverse curves.

\subsection{Final balancer of lateral tanks fill levels}

The task of the final balancer is to re-establish equality between the two lateral tanks fill levels once that all the other regulators stop and external lateral acceleration becomes zero, i.e., the curve is over. Its intervention can be easily described through the following expressions:

$$
\text { If : } Q_{\text {comp1 }}=Q_{\text {comp2 }}=Q_{\text {threshold }}=0 \frac{m^{3}}{\mathrm{~s}}, \frac{|\Delta V|}{V_{l}} \geq 1.63 \%, \quad-0.01 \leq \ddot{x} \leq 0.01 \frac{\mathrm{m}}{\mathrm{s}^{2}}
$$

then $|Q|>0 \frac{m^{3}}{S}, \operatorname{sign}(Q)=-\operatorname{sign}(\Delta V)$.

Therefore, during the first straight stretch of road after cornering, the final balancer commands the electropumps to move a liquid flow to the emptier compensation tank until the relative difference $|\Delta V| / V_{1}$ (where $V_{1}$ is the total capacity of one lateral tank) between the volumes of fluid inside the lateral tanks exceeds $1.63 \%$. It is necessary to introduce a threshold regarding the difference between the two volumes to prevent an alternate flow rate without ever reaching the perfect equality of fill levels, but tending to it.

\section{Tank layout optimisation}

\subsection{Standard configurations comparison}

The first step in evaluating the lateral stability improvement achieved with the presented anti-rollover system is the definition of the best standard tanker truck configuration 
in terms of lower lateral load transfer while cornering. First of all, a lateral acceleration input, for the comparison of the various configurations behaviour while cornering, must be defined. It is assumed that lateral acceleration varies linearly from zero to a maximum of $5 \mathrm{~m} / \mathrm{s}^{2}$ in five seconds; then, the maximum acceleration remains constant for $7 \mathrm{~s}$ before gradually returning to the value of $0 \mathrm{~m} / \mathrm{s}^{2}$ following the same trend assumed for the acceleration rising. This is to simulate driving during a gradually negotiated curve. A set of standard tanker trucks, with aspect ratios of the tank section varying from $a / b=1$ (circular tank section) to $a / b=2$, has been defined, all the standard tanker trucks are characterised by identical maximum liquid cargo capability and identical transported liquid load. Dimensions of the tanks and fill levels, to obtain equality between the transported and the transportable liquid volumes, have been calculated by means of Matlab. Tank section base point is located at the same position (relative to the coordinate system shown in Figures 1 and 2) for all the examined standard tank truck configurations, which means that a constant ground clearance has been assumed. Ultimately, the comparison between all the configurations is affected considering all equal conditions. Figure 3 shows all the examined tank layouts and their corresponding main parameters. Figure 4 shows the simulation results obtained by applying the above-mentioned acceleration input to the five configurations in exam. Despite the vehicle model is only valid if $|R| \leq 1$, for the sake of comparability, the simulations are continued until the end of the manoeuvre. As can be observed in Figure 4, considering partially filling conditions, equal transported liquid load and maximum liquid cargo capability, the best lateral stability is obtained when the tank has a circular cross section. This is due to the fact that, despite the lower static CG position that characterises the elliptical configurations, the sloshing mass causes a CG lateral shift that is greater for higher cross-section aspect ratios, which means for gradually more elliptical cross sections. Obviously, the situation is reversed under full load conditions, being the lateral stability completely defined by the static CG position. Anti-rollover system performances will be evaluated through the comparison of lateral stability achieved by tanker trucks equipped with it and by standard tanker trucks having circular cross section.

Figure 3 Tanker trucks configurations (active 3 layout includes small additional elliptical tanks)

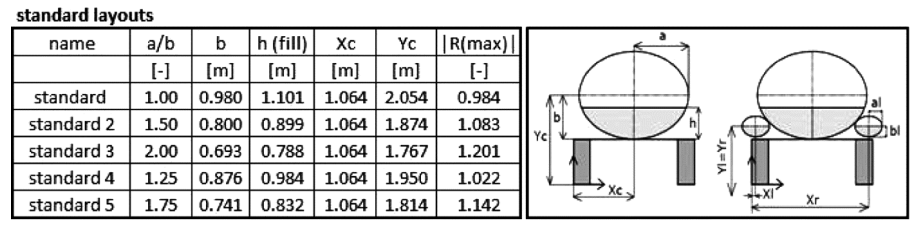

active layouts
\begin{tabular}{|c|c|c|c|c|c|c|c|c|c|c|c|c|}
\hline name & $\mathrm{a} / \mathrm{b}$ & $\mathrm{b}$ & $\mathrm{h}$ (fill) & $\mathrm{Xc}$ & $\mathrm{Yc}$ & $\mathrm{XI}$ & $\mathrm{Xr}$ & $\mathrm{Yr}=\mathrm{Yl}$ & $\mid \mathrm{R}(\mathrm{max})$ & $\mathrm{a}_{/} / \mathrm{b}_{1}$ & $\mathrm{~b}_{1}$ & $\mathrm{Q}$ \\
\hline & {$[-]$} & {$[\mathrm{m}]$} & {$[\mathrm{m}]$} & {$[\mathrm{m}]$} & {$[\mathrm{m}]$} & {$[\mathrm{m}]$} & {$[\mathrm{m}]$} & {$[\mathrm{m}]$} & {$[-]$} & {$[\mathrm{m}]$} & {$[\mathrm{m}]$} & {$\left[\mathrm{m}^{3} / \mathrm{s}\right]$} \\
\hline active 1 & 1.00 & 0.900 & 0.900 & 1.064 & 2.170 & -0.016 & 2.144 & 1.472 & 0.852 & 1.00 & 0.3875 & 0.200 \\
\hline active 2 & 1.00 & 0.929 & 0.973 & 1.064 & 1.997 & -0.066 & 2.194 & 1.472 & 0.844 & 1.00 & 0.3125 & 0.125 \\
\hline active 3 & 1.00 & 0.918 & 0.946 & 1.064 & 1.986 & -0.066 & 2.194 & 1.472 & 0.828 & 1.00 & 0.3125 & 0.125 \\
\hline ellipse & 1.25 & 0.831 & 0.870 & 1.064 & 2.029 & -0.066 & 2.194 & 1.397 & 0.929 & 1.00 & 0.3125 & 0.125 \\
\hline active 4 & 1.00 & 0.931 & 0.978 & 1.064 & 2.083 & -0.016 & 2.144 & 1.410 & 0.853 & 1.50 & 0.2500 & 0.200 \\
\hline active 5 & 1.00 & 0.931 & 0.978 & 1.064 & 2.077 & -0.016 & 2.144 & 1.434 & 0.852 & 1.25 & 0.2740 & 0.190 \\
\hline ellipse 2 & 1.25 & 0.831 & 0.870 & 1.064 & 2.029 & -0.074 & 2.196 & 1.374 & 0.934 & 1.25 & 0.2800 & 0.125 \\
\hline
\end{tabular}

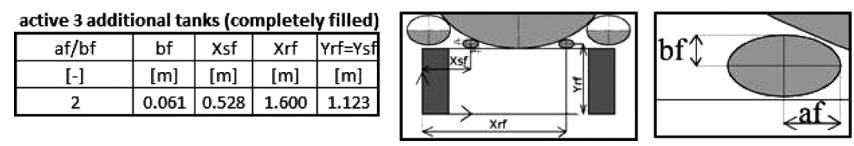


Figure 4 Standard tanker trucks: lateral load transfer comparison

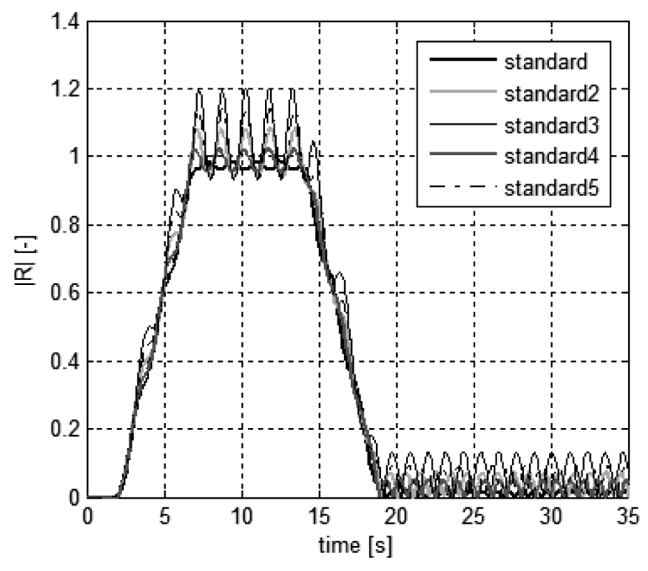

\subsection{Layout optimisation}

The aim of the layout optimisation is the achievement of a significant improvement in lateral stability of the vehicle, along with a contemporary minimisation of the needed flow rate necessary to move from one lateral tank to the other. This means reducing the number of needed pumps, the space necessary for the positioning of the latter and the ensuing extra weight involved. Optimisation of shape and placement of the tanks also means allowing a satisfactory reduction of rollover risk with realisable tanker layouts, i.e., layouts not involving evident restrictions on the suspensions kinematics or the use of tyres with an excessively reduced diameter. As before, the comparison between the 'active' (i.e., tanker trucks equipped with the anti-rollover system) tanker layouts, while cornering, is performed at equal conditions. Specifically, the acceleration input is the same as that used for the standard configurations comparison and all the active tanker trucks are characterised by identical maximum liquid cargo capability and transported liquid load. Main tank fill levels and dimensions for each layout have been calculated by means of Matlab. Filling percentage is constant for all simulations reported in Sections 5.1 and 5.2. Several 'active' tanker layouts (Figure 3 lists some of them) have been schematically designed and simulations results analysed. Figure 5 shows simulation results for some interesting layouts and for the standard circular tanker truck. Thanks to layout optimisation, the flow rate required to achieve significant improvements in terms of lateral load transfer reduction, changed from $0.2 \mathrm{~m}^{3} / \mathrm{s}$ (for the first layout, activel) to $0.125 \mathrm{~m}^{3} / \mathrm{s}$ (for the most stable configurations, i.e., active 2 and active3). Note that lateral stability improvements achieved by active 2 and active3 layouts are strictly similar to the performance obtained by activel configuration as you can see in Figure 5, but with a significant reduction of required flow rate. Although its benefits are nothing impressive, for the sake of completeness, Figure 5 also shows the simulation results corresponding to a layout with elliptical main tank (ellipse). 
Figure 5 Active tanker trucks: lateral load transfer comparison

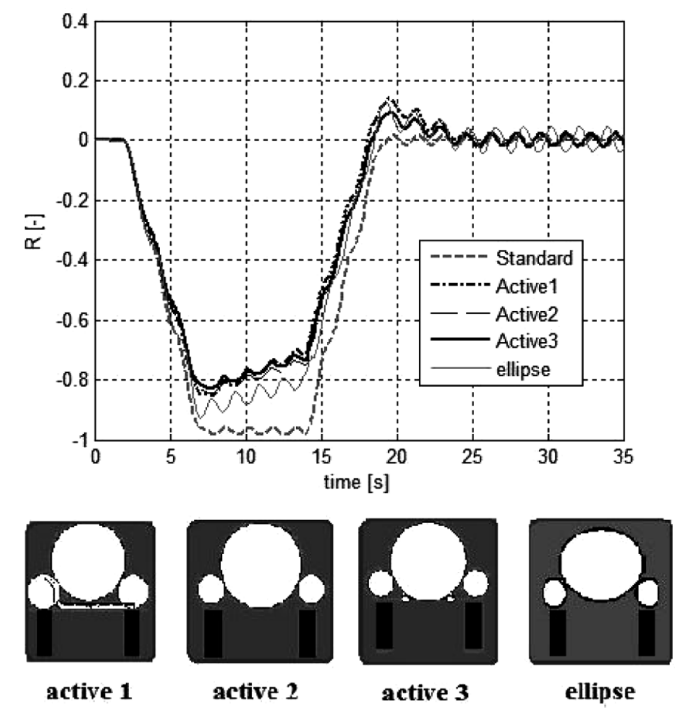

\section{Power absorption braking effect}

Every modern motorist has experienced the power absorption effect and the resulting slowing down occurring every time the air-conditioning system is activated. A similar effect takes place for tanker trucks equipped with the anti-rollover system whenever the pumps are working. The slowing down that occurs every time the pumps are working to transfer liquid from one lateral tank to the other is an integral part of the anti-rollover system, as it contributes on reducing lateral acceleration while cornering. The benefit provided by this braking effect, although slight, is, of course, the more significant the longer is the working time.

The braking effect due to pumps power absorption is simulated assuming that the driver provides, through the accelerator pedal, the power required for uniform motion at a certain speed. Traction force required for motion at constant speed is the force necessary to win aerodynamic drag, tyres rolling resistance and the component of weight along the direction of motion. Thus, in the most general case, power required for uniform motion is determined by the following equation (Genta, 2000) (see Nomenclature):

$$
P_{n}(V)=V \cdot R_{d r a g}=A \cdot V+B \cdot V^{3}+C \cdot V^{5}
$$

where (see Table 1 for vehicle parameters):

$$
\begin{aligned}
& A=m_{\mathrm{tot}} \cdot g \cdot\left[f_{o} \cdot \cos (\alpha)+\sin (\alpha)\right] \\
& B=m_{\mathrm{tot}} \cdot g \cdot k \cdot \cos (\alpha)+\frac{1}{2} \cdot \rho \cdot S \cdot\left[C_{x}-C_{z} \cdot f_{o}\right] \\
& C=-\frac{1}{2} \cdot \rho \cdot S \cdot k \cdot C_{z}
\end{aligned}
$$




$$
S=\psi \cdot w \cdot h_{1}
$$

and $R_{\text {drag }}$ is the total drag to vehicle motion. Once the liquid transfer from one lateral tank to the other has started, power to wheels must be reduced of the power $P_{\text {absorp }}$ needed to operate the pump, with a resulting power deficit. Consequently, power to wheels becomes less than that required for uniform motion and a gradual slight reduction of speed occurs according to the following equation:

$$
\frac{\mathrm{d} V}{\mathrm{~d} t}=\frac{1}{m_{\mathrm{tot}}} \cdot \frac{\Delta P(V)}{V}
$$

where

$$
\begin{aligned}
& \Delta P(V)=P_{\text {prov }}-P_{n}(V) \\
& P_{\text {prov }}=P_{n}(V)-P_{\text {absorp }} .
\end{aligned}
$$

Once the pumps have stopped working, power to wheels grows again and vehicle speed gradually increases tending over time to the initial value.

Table 1 Vehicle parameters

\begin{tabular}{lcc}
\hline Parameters & Value & Units \\
\hline$f_{o}$ & 0.008 & {$[-]$} \\
$k$ & 0 & {$\left[\mathrm{~s}^{2} / \mathrm{m}^{2}\right]$} \\
$C_{x}$ & 0.65 & {$[-]$} \\
$C_{z}$ & 0 & {$[-]$} \\
$\psi$ & 0.95 & {$[-]$} \\
$w$ & 2.128 & {$[\mathrm{~m}]$} \\
$h_{1}$ & 3.803 & {$[\mathrm{~m}]$} \\
$m_{\text {tot }}$ & 26424 & {$[\mathrm{~kg}]$} \\
$\rho$ & 1.2258 & {$\left[\mathrm{~kg} / \mathrm{m}^{3}\right]$} \\
\hline
\end{tabular}

\section{Coupling between longitudinal and lateral fluid sloshing}

In the case of inclined roads or if the tanker truck rapidly varies its speed (heavy braking or harsh acceleration), the free surface of the liquid contained within the tanks does not remain parallel to the longitudinal axis of the tanker truck but it is positioned in such a way as to maintain itself approximately orthogonal to the resultant of force of gravity and force of inertia. Because, as discussed in Section 3, all the trammel pendulum parameters depend on the fill levels of the tanks, the dynamics of longitudinal liquid movement affects the dynamics of lateral fluid sloshing and, consequently, the vehicle lateral stability. This is easily understandable if one imagines to run along a particularly inclined stretch of road or to make a sudden braking. In these cases, lateral liquid sloshing 
is characterised by a higher fill level compared with the fill level corresponding to uniform motion on a horizontal road. Coupling between longitudinal and lateral fluid sloshing is, at a parity of inclination of free liquid surface, more important for lateral tanks than the main tank. This is because, although longitudinal dimensions of all tanks are the same, lateral tanks cross section has a lower vertical dimension.

An algorithm has been developed to calculate the inclination $\alpha^{\prime}$ of the free surface of the liquid as (Figure 6):

$$
\tan \left(\alpha^{\prime}\right)=\frac{\mathrm{d} V / \mathrm{d} t+g \cdot \operatorname{sen}(\alpha)}{g \cdot \cos (\alpha)}=\tan (\alpha)+\frac{\mathrm{d} V / \mathrm{d} t}{\mathrm{~g} \cdot \cos (\alpha)}
$$

where $\alpha$ is the longitudinal inclination of the road surface (positive in the case of climb), $V$ is the vehicle speed and $g$ is the gravitational acceleration. Then, through an iterative process of discretisation and calculation of the transported liquid volume, it determines a very good approximation of the liquid disposition within the tank. Finally, it provides the fill level to consider in case of inclined roads or strong accelerations (as weighted average of the fill levels corresponding to the fractions of fluid in which the transported liquid volume is discretised) according to the following equations (Figure 7):

$$
\begin{aligned}
& h_{\text {incl }}=\frac{\sum_{1}^{10} V h(i) \cdot h(i)+\left[\left(z_{1}-\left(-\frac{L}{2}\right)\right) \cdot \pi \cdot a \cdot b\right] \cdot 2 b}{V_{\mathrm{CALC}}} \text { if } \tan \left(\alpha^{\prime}\right)<0 \\
& h_{\text {incl }}=\frac{\sum_{1}^{10} V h(i) \cdot h(i)+\left[\left(\frac{L}{2}-z_{2}\right) \cdot \pi \cdot a \cdot b\right] \cdot 2 b}{V_{\mathrm{CALC}}} \text { if } \tan \left(\alpha^{\prime}\right)>0
\end{aligned}
$$

where $V h(i)$ is the volume $\left[\mathrm{m}^{3}\right]$ of the $i$ th fraction of fluid in which the transported liquid volume is discretised, $h(i)$ is the fill level corresponding to the latter, $L$ is the length of the tank ( $L=10 \mathrm{~m}$ for all considered tanks), $z_{1}$ and $z_{2}$ are the longitudinal coordinates where the free surface of the liquid touches the contour of the tank and $V_{\text {CALC }}$ is the total liquid volume transported inside the tank, as calculated by the algorithm while $a$ and $b$ are described in Nomenclature.

Figure 6 Liquid position within the tank during downhill braking

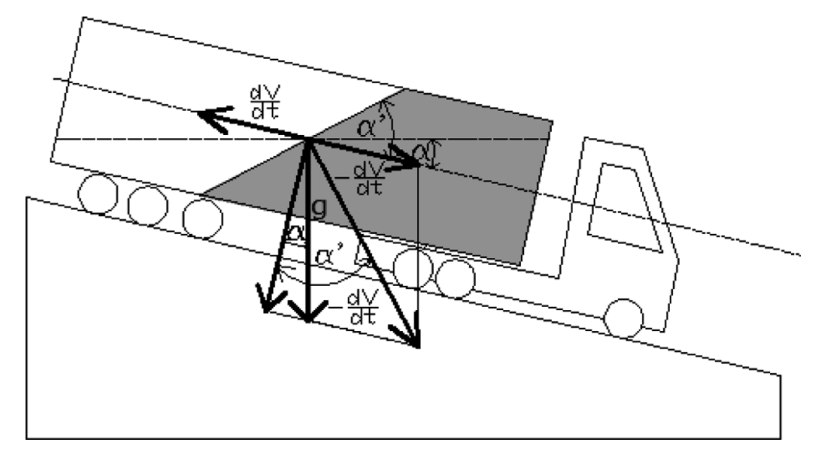


Figure 7 Discretised longitudinal position of the liquid inside a tank

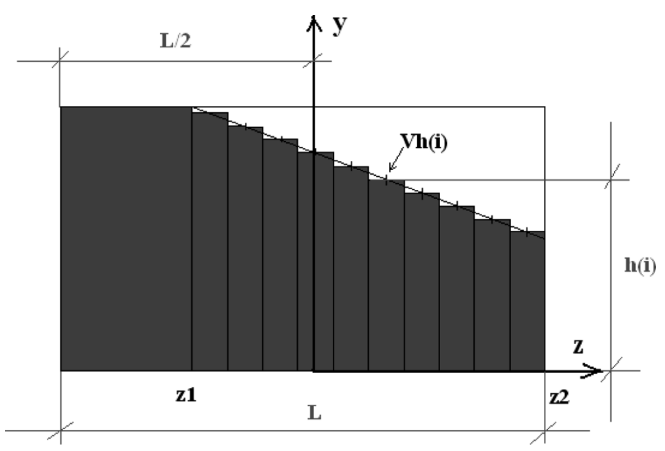

\section{Simulation examples}

Many simulations have been developed to study 'active' tanker trucks behaviour while cornering. This section describes the system response for some representative vehicle manoeuvres. Rollover risk reduction performances, in terms of lateral stability improvement, are well evaluated when the tanker truck is negotiating an S-shaped curve (Figure 8). Two simulations for two different S-shaped curves have been performed. It is important to examine the anti-rollover system response for a succession of reverse curves to demonstrate the system ability to react promptly, restoring the equality of the lateral tanks fill levels and filling the inner lateral tank before the second curve has occurred.

To guarantee a gradual variation of lateral acceleration and a correct visual perception of the bending of the road, a variable-radius curve is used for the transition between a straight stretch of road and a constant radius curve (like, for example, in highway exits). Such a kind of transition curve belongs to the family of spirals and is named clotoidal transition. A clotoidal transition is defined by the following equation:

$$
r \cdot s=A_{c l}^{2}
$$

where $r$ is the radius of curvature at the current point, $s$ is the curvilinear abscissa at the current point and $A_{c l}$ is the clotoidal transition parameter.

Figure 8 Simulation 1 and Simulation 2 curves ( ${ }^{*}$ ' $=$ clotoidal transitions start points, 'o' = clotodail transitions end points)
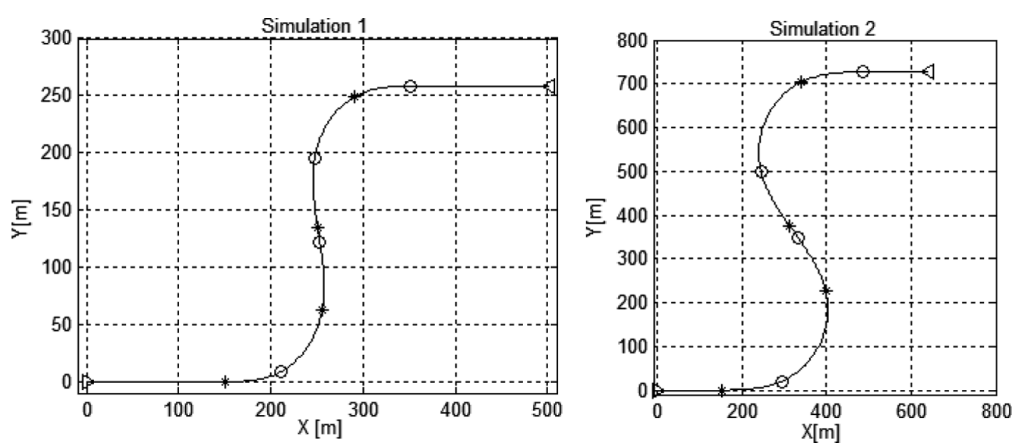
According to normative (Italian Ministry of Infrastructure and Transport, 2002) concerning the building of roads, in the case of S-shaped curves, it is possible to interpose between the curves a brief straight stretch of road having a length not exceeding:

$$
L_{\text {sim }}=\frac{A_{c l 1}+A_{c l 2}}{12.5}
$$

where $A_{c l 1}$ and $A_{c l 2}$ are, respectively, the clotoidal transition parameters for the two subsequent curves.

In simulation 1, the curves (Figure 8) have an opening of $55^{\circ}$ and a minimum radius of $77 \mathrm{~m}$, while the clotoidal transition parameter is equal to $A_{c l 1}=70 \mathrm{~m}$. In simulation 2, the curves have an opening (Figure 8 ) of $80^{\circ}$ and a minimum radius of $180 \mathrm{~m}$, being the clotoidal transition parameter equal to $A_{c l 2}=164 \mathrm{~m}$. Two brief straight stretches of road with lengths equal to:

$$
L_{\text {sim } 1}=\frac{2 \cdot A_{c l 1}}{12.5}=11.2 \mathrm{~m} \quad L_{\text {sim } 2}=\frac{2 \cdot A_{c l 2}}{12.5}=26.64 \mathrm{~m}
$$

are interposed between the two successive curves, respectively, for simulations 1 and 2 . Tanker trucks initial speed is equal to $19.44 \mathrm{~m} / \mathrm{s}(70 \mathrm{~km} / \mathrm{h})$ for simulation 1 and to $30 \mathrm{~m} / \mathrm{s}$ $(108 \mathrm{~km} / \mathrm{h})$ for simulation 2 . While speed is constant for the standard circular tanker truck, a slight and gradual speed reduction due to the pumps, power absorption occurs for tanker trucks equipped with the proposed anti-rollover system. As you can see, speed is excessive for both the simulated S-shaped curves. This is because simulations are representative of all cases in which the driver proceeds at a too high speed because of an incorrect optical perception of the constant radius curve after the clotoidal transition. Figures 9 and 10 show the responses of the conventional circular tanker truck and of the 'active' tanker trucks when a lateral acceleration input, corresponding to simulation 1 and simulation 2 , is applied. Notice that tanker trucks equipped with the anti-rollover system obtain improvements in terms of lateral load transfer. In simulation 1, the maximum absolute value of $R$, the normalised lateral load transfer, for the standard circular tanker truck is $\left|R_{\max \text { std }}\right|=0.9$, which is obtained while negotiating the second curve, while active 2 and active3 layouts achieve $\left|R_{\text {max_act } 2}\right|=0.794$ and $\left|R_{\text {max } \_ \text {act } 3}\right|=0.783$, respectively. In simulation 2, the maximum absolute value of $R$ for the standard tanker truck is $\left|R_{\max \text { std }}\right|=0.913$, while active 2 and active 3 configurations obtain $\left|R_{\text {max } \_ \text {act } 2}\right|=0.806$ and $\left|R_{\text {max } \_ \text {act3 }}\right|=0.796$. These results are achieved thanks to the simultaneous action of all stabilising effects, i.e. the active contribution provided by the controllers and the passive contributions provided by the CG lowering and by the braking effect due to the pumps power absorption. As can be seen in Figures 11 and 12, liquid flow between the two lateral tanks faithfully follows the lateral acceleration input. Moreover, left lateral tank volume filling over time is such that, when the second curve point of maximum curvature is reached, the inner lateral tank is, in any case, fuller than the outer lateral tank. Figure 12 also shows that flow rate to the inner lateral tank stops after $5 \mathrm{~s}$ from the moment in which the external lateral acceleration stops increasing. 
Figure 9 Simulation 1 results: normalised lateral load transfer vs. time

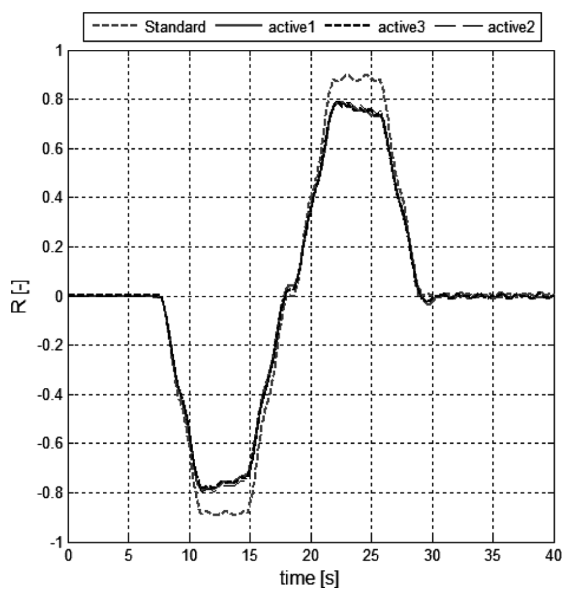

Figure 10 Simulation 2 results: normalised lateral load transfer vs. time

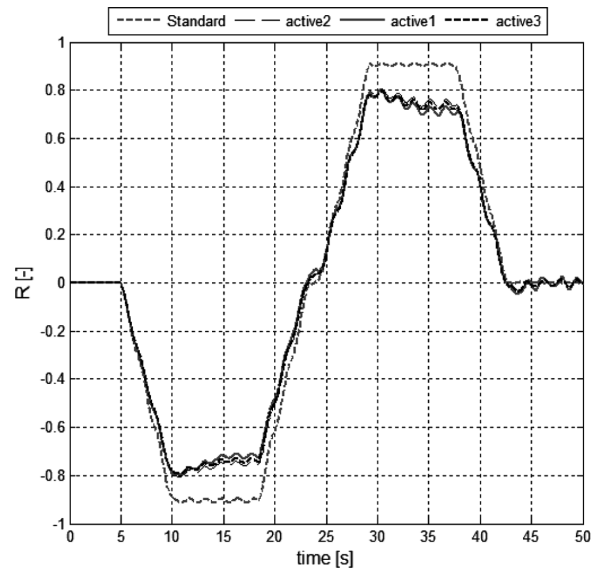

Figure 11 Simulation 1: acceleration, flow rate and left lateral tank liquid volume vs. time
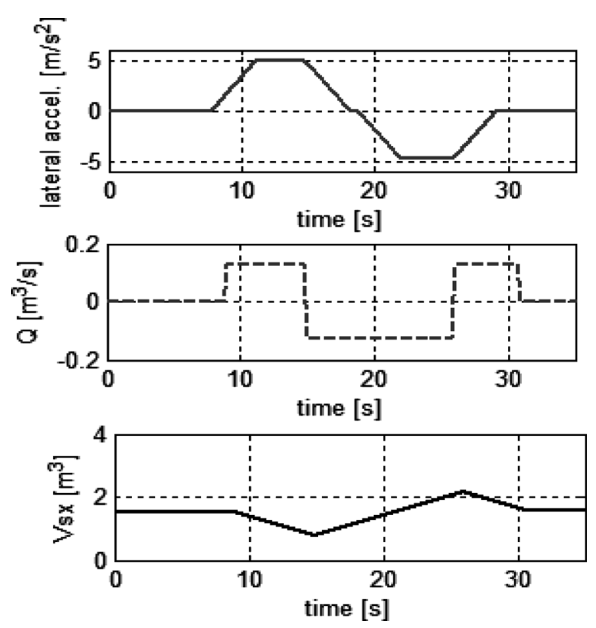
Figure 12 Simulation 2: acceleration, flow rate and left lateral tank liquid volume vs. time
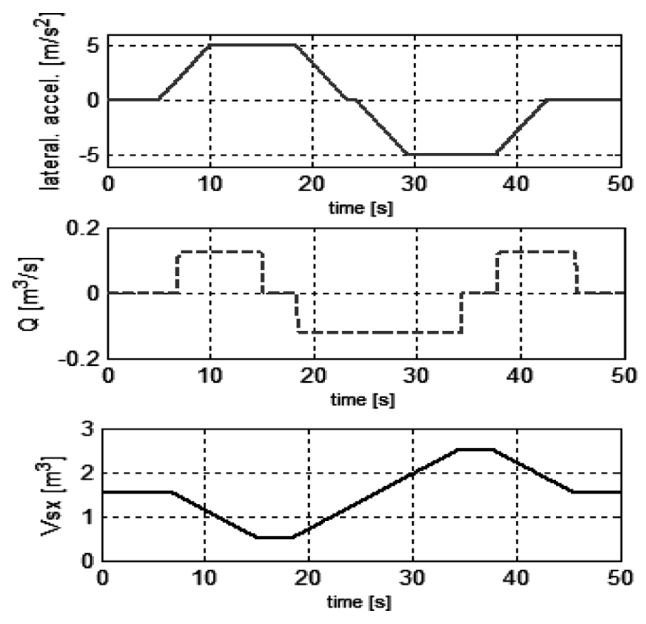

Figure 13 shows the comparison between the performances obtained by the 'active' tanker trucks and by the standard circular tanker truck during a downhill curve (simulation 3). Simulation 3 analyses a 180 degree curve (hairpin turn) with a minimum radius of curvature of $45 \mathrm{~m}$ and a clotoidal transition parameter equal to $A_{c / 3}=30 \mathrm{~m}$. The curve is all downhill (horizontal and variable slope stretches of road are external to the curve) with a slope equal to $5.7^{\circ}(10 \%)$. Tanker trucks initial speed is $40 \mathrm{~km} / \mathrm{h}$ $(11.11 \mathrm{~m} / \mathrm{s})$. Braking does not allow uniform motion, thus speed continuously increases. Consequently, the slowing effect due to pumps power absorption plays a key role in rollover avoidance and lateral load transfer reduction. As shown in the diagram, even in this case tanker trucks equipped with the anti-rollover system achieve significant improvements in terms of lateral load transfer. Coupling between longitudinal and lateral fluid sloshing has also been taken into account.

Figure 13 Simulation 3 results: normalised lateral load transfer vs. time

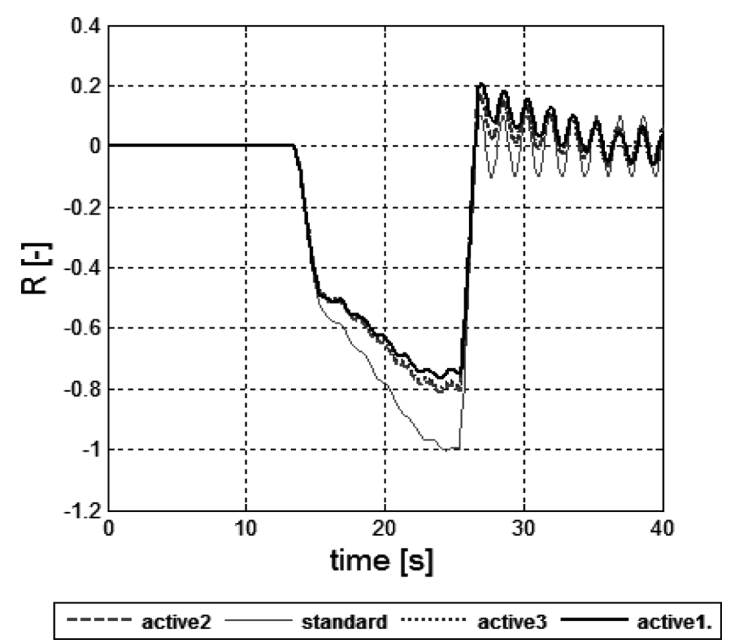




\section{Conclusions}

In this paper, a new anti-rollover system for tanker trucks that directly acts on the lateral distribution of the transported liquid cargo has been proposed. The system consists of a main tank and two smaller lateral tanks, which can change their fill levels thanks to the employment of a set of electropumps suitable for flammable liquids pumping. Liquid lateral sloshing dynamics is simulated using trammel pendulums. Liquid transfer from one lateral tank to the other is controlled by a set of feedback regulators. Several simulations have been performed and examined. Thanks to the simultaneous action of active and passive stabilising contributions, the system provides significant improvements in terms of lateral load transfer while cornering. In all performed simulations, active tanker trucks' lateral stability performances are better than those obtained by standard tanker trucks.

\section{References}

Acarman, T. and Özgüner, U. (2003) 'Rollover prevention for heavy trucks using frequency shaped sliding mode control', Proceedings of IEEE Conference on Control Applications, Istanbul, Turkey, Vol. 1, June, pp.7-12.

Bolzern, P., Scattolini, R. and Schiavoni, N. (2004) Fondamenti di Controlli Automatici, McGraw-Hill, Italia, Milano.

Citrini, D. and Noseda, G. (1987) Idraulica, Casa Editrice Ambrosiana (CEA), Milano.

Dodge, F.T. (2000) 'The new dynamic behavior of liquids in moving containers', Southwest Research Institute, Stan Antonio, Texas, pp.1-16.

Eisele, D. (2000) 'Vehicle dynamics control with rollover prevention for articulated heavy trucks', Proc. AVEC 2000 5th Int. Symposium on Advanced Vehicle Control, 22-24 August, Ann Arbour, Michigan.

Gáspár, P., Szabó, Z. and Bokor, J. (2005) 'Prediction based combined control to prevent the rollover of heavy vehicles', Proc. 13th Mediterranean Conference on Control and Automation, 27-29 June, Limassol, Cyprus, pp.575-580.

Genta, G. (2000) Meccanica dell'autoveicolo, Levrotto e Bella, Torino.

Hac, A. (2000) 'Influence of Active Chassis Systems on Vehicle Propensity to Maneuver-Induced rollovers', Proc. SAE 2002 World Congress, 4-7 March, Detroit, Michigan, SAE paper 2002-01-0967.

Ministero delle Infrastrutture e dei Trasporti (Italian Ministry of Infrastructure and Transport) (2002) 'Norme funzionali e geometriche per la costruzione delle strade', supplemento ordinario alla gazzetta ufficiale, serie generale n.3, 4 January, Rome, Italy.

Odenthal, D., Bünte, T. and Ackermann, J. (1999) 'Nonlinear steering and brake control for vehicle rollover avoidance', European Control Conference, CD-ROM, Karlsruhe, Germany, http://citeseer.ist.psu.edu

Salem, M.I. (2000) Rollover Stability of Partially Filled Heavy Duty Elliptical Tankers using Trammel Pendulum to Simulate Fluid Sloshing, PhD Thesis, West Virginia University, https://eidr.wvu.edu/files/1239/Salem_M_ETD.pdf

Sampson, D.J.M. (2000) Active Roll Control of Articulated Heavy Vehicles, PhD Thesis, Cambridge University Engineering Department, http://david.sampson.id.au/vehicle/phd.html

Sampson, D.J.M., Jeppesen, B.P. and Cebon, D. (2000) 'The development of an active roll control system for heavy vehicles', Proc. 6th Int. Symp. on Heavy Vehicle Weights and Dimensions, Saskatoon, Canada, , pp.375-384. 
Winkler, C. (2000) 'Rollover of commercial heavy vehicles', UMTRI Research Review, University of Michigan Transportation Research Institute, Vol. 31, No. 4, pp.1-17.

\section{Nomenclature}

\begin{tabular}{|c|c|c|}
\hline Symbols & Description & Units \\
\hline$a$ & Horizontal semiaxis of the tank elliptical section & {$[\mathrm{m}]$} \\
\hline$b$ & Vertical semiaxis of the tank elliptical section & {$[\mathrm{m}]$} \\
\hline $\bar{a}$ & Major axis of the trammel pendulum & {$[\mathrm{m}]$} \\
\hline $\bar{b}$ & Minor axis of the trammel pendulum & {$[\mathrm{m}]$} \\
\hline$M_{t}$ & Total fluid mass & {$[\mathrm{kg}]$} \\
\hline$M_{p}$ & Sloshing fluid mass & {$[\mathrm{kg}]$} \\
\hline$M_{f}$ & Fixed portion of fluid mass & {$[\mathrm{kg}]$} \\
\hline$G$ & Gravitational acceleration & {$\left[\mathrm{m} \mathrm{s}^{2}\right]$} \\
\hline$\vartheta$ & Trammel pendulum angle of swing & {$[\mathrm{rad}]$} \\
\hline$H$ & Tank fill level & {$[\mathrm{m}]$} \\
\hline$\ddot{x}$ & External lateral acceleration & {$\left[\mathrm{m} \mathrm{s}^{2}\right]$} \\
\hline$\ddot{r_{x}}$ & Horizontal component of moving mass acceleration & {$\left[\mathrm{m} \mathrm{s}^{2}\right]$} \\
\hline$\ddot{r}_{y}$ & Vertical component of the moving mass acceleration & {$\left[\mathrm{m} \mathrm{s}^{2}\right]$} \\
\hline$F_{p 1, x}$ & Horizontal component of the moving mass inertial force & {$[\mathrm{N}]$} \\
\hline$F_{p 1, y}$ & Vertical component of the moving mass inertial force & {$[\mathrm{N}]$} \\
\hline$F_{p 2, y}$ & Weight of the fluid sloshing mass & {$[\mathrm{N}]$} \\
\hline$F_{f, x}$ & Fixed mass centrifugal force & {$[\mathrm{N}]$} \\
\hline$F_{f, x}$ & Weight of the fixed fluid mass & {$[\mathrm{N}]$} \\
\hline$F_{x, R}$ & Right wheels vertical load & {$[\mathrm{N}]$} \\
\hline$F_{x, L}$ & Left wheels vertical load & {$[\mathrm{N}]$} \\
\hline$R$ & Normalised lateral load transfer & {$[-]$} \\
\hline$V$ & Vehicle speed & {$[\mathrm{m} / \mathrm{s}]$} \\
\hline$P_{n}(V)$ & Power required for uniform motion & {$[\mathrm{W}]$} \\
\hline$m_{\text {tot }}$ & Total vehicle mass & {$[\mathrm{kg}]$} \\
\hline$f_{\mathrm{o}}$ & Tyres rolling resistance coefficient at $V=0$ & {$[-]$} \\
\hline$K$ & Rolling resistance coefficient & {$\left[\mathrm{s}^{2} / \mathrm{m}^{2}\right]$} \\
\hline$C_{\mathrm{x}}$ & Aerodynamic drag coefficient & {$[-]$} \\
\hline$C_{\mathrm{z}}$ & Aerodynamic lift coefficient & {$[-]$} \\
\hline$S$ & Vehicle frontal area & {$\left[\mathrm{m}^{2}\right]$} \\
\hline$\psi$ & Corrective coefficient for vehicle frontal area & {$[-]$} \\
\hline$w$ & Vehicle track width & {$[\mathrm{m}]$} \\
\hline$h_{1}$ & Vehicle height & {$[\mathrm{m}]$} \\
\hline$\rho$ & Air density & {$\left[\mathrm{kg} / \mathrm{m}^{3}\right]$} \\
\hline
\end{tabular}

